\title{
Sweyjawbu expression is a predictor of ALK rearrangement status in lymphoma
}

\author{
Kai Xue ${ }^{1,4, *}$, Xun $\mathrm{Ye}^{2,5, *}$, Fang Liư ${ }^{2}$, Qunlin Zhang ${ }^{1,4}$, Qifeng Wang ${ }^{3,4}$, Shan Huang ${ }^{6}$, \\ Jiachen Wang ${ }^{1,4}$, YongMing Lu ${ }^{3,4}$, Ye Guo ${ }^{1,4}$, Xia Meng ${ }^{2,5}$ \\ ${ }^{1}$ Department of Medical Oncology, Fudan University Shanghai Cancer Center, Shanghai, China \\ ${ }^{2}$ Fudan University Shanghai Cancer Center, Institut Mérieux Lab, Fudan University Shanghai Cancer Center, Shanghai, China \\ ${ }^{3}$ Department of Pathology, Fudan University Shanghai Cancer Center, Shanghai, China \\ ${ }^{4}$ Department of Oncology, Shanghai Medical College, Fudan University, Shanghai, China \\ ${ }^{5}$ Medical Device Development Department (MD3), bioMérieux Co., Ltd., Shanghai, China \\ ${ }^{6}$ Department of Pathology, The First Affiliated Hospital of Soochow University, Suzhou, China \\ *These authors have contributed equally to this work. \\ Correspondence to: Ye Guo, email: pattrickguo@gmail.com \\ Xia Meng, email: xmeng00@hotmail.com
}

Keywords: sweyjawbu, ALK rearrangements, lymphoma

Received: February 24, $2016 \quad$ Accepted: November 02, $2016 \quad$ Published: December 10, 2016

\section{ABSTRACT}

In recent years molecular subtyping has become an important tool for accurate diagnosis of many cancers; for example, the detection of ALK rearrangements in lymphoma and lung cancer helps clinicians provide more precise diagnosis and treatment. Fluorescence in situ hybridization (FISH) and immunohistochemistry (IHC) are two routine approaches used to detect ALK rearrangements. However, difficulties with acquisition of biopsy samples, high costs, and long waiting time for results negatively impact the application of these methods. A rapid and inexpensive alternative would be a useful complement to current ALK rearrangement detection. We identified a novel gene, sweyjawbu, from Affymetrix microarray studies. Its expression correlated strongly with ALK in an analysis of 1037 cancer cell lines (correlation coefficient $=0.92$ ). By comparing sweyjawbu transcript levels, it was possible to discriminate 12 ALK rearrangement-positive lymphoma samples from 64 ALK rearrangement-negative lymphomas. Moreover, combining measurements of sweyjawbu expression and the ratio of the 5' and 3' portions of the ALK transcript provided even more accurate identification of ALK rearrangement-positive lymphomas. This novel approach is an excellent complement or alternative to existing FISH and IHC methodologies.

\section{INTRODUCTION}

Lymphoma is one of ten most common malignancies worldwide, and chemotherapy is the backbone for its treatment. Highly aggressive relapsed and refractory lymphomas remain a major challenge for clinicians $[1,2]$. Genetic alterations commonly cause constitutive activation of kinases in lymphomas. Anaplastic lymphoma kinase (ALK), also known as ALK tyrosine kinase receptor or CD246 (cluster of differentiation 246), is an enzyme encoded by the human ALK gene [3]. Functional chimeric proteins formed by fusing ALK with other proteins have found in many lymphoma subtypes [4], including anaplastic large-cell lymphoma (ALCL) and diffuse large B-cell lymphoma (DLBCL). With the development of precision medicine, detection of ALK rearrangements are now required in new diagnosis of ALCL and may also be considered in DLBCL [5]. Crizotinib, an inhibitor of tyrosine receptor kinases, including ALK and CD117, has been found to be effective against inflammatory myofibroblastic tumor or non-small cell lung cancer with ALK rearrangement [6]. Successful applications of the 
drug on the target protein/gene in ALK rearrangementpositive lymphoma have also been reported [7, 8]. FISH and/or IHC are currently used to characterize ALK rearrangement status. However, these techniques require tissue samples which are difficult to obtain from specific lymphatic disease patients.

The widely used Affymetrix U133 Plus 2.0 microarray allows us to test expression of thousands of genes simultaneously, including both coding and non-coding RNA. With this microarray, Piva et al. compared the expression profile from 16 ALCL samples with confirmed ALK rearrangement and 20 ALK rearrangement-negative ALCL samples and selected probe set 242964_at as one of the most significant differentially expressed genes, with a 47.58 fold change. Probe set 242964_at corresponds to sweyjawbu RNA, determined after aligning its probe sequences to an AceView RNA sequence collection database (www.ncbi.nlm.nih.gov/ ieb/research/acembly) [9, 10]. We characterized the expression of sweyjawbu in a number of lymphoma cell lines in order to evaluate its usefulness as a predictor of ALK rearrangement status.

\section{RESULTS}

\section{sweyjawbu expression is highly upregulated in ALK rearrangement-positive cell lines}

The Cancer Cell Line Encyclopedia (CCLE) database provided analysis results of mRNA expression for 1037 cancer cell lines (www.broadinstitute.org/ccle) [9]. sweyjawbu RNA expression is associated with ALK gene amplification or translocation status in a number of different types of cancer cells. The correlation coefficient between signal intensity of probe sets 208212 _s_at and 242964_at in 1037 cancer cells reached 0.92 (Figure 1).
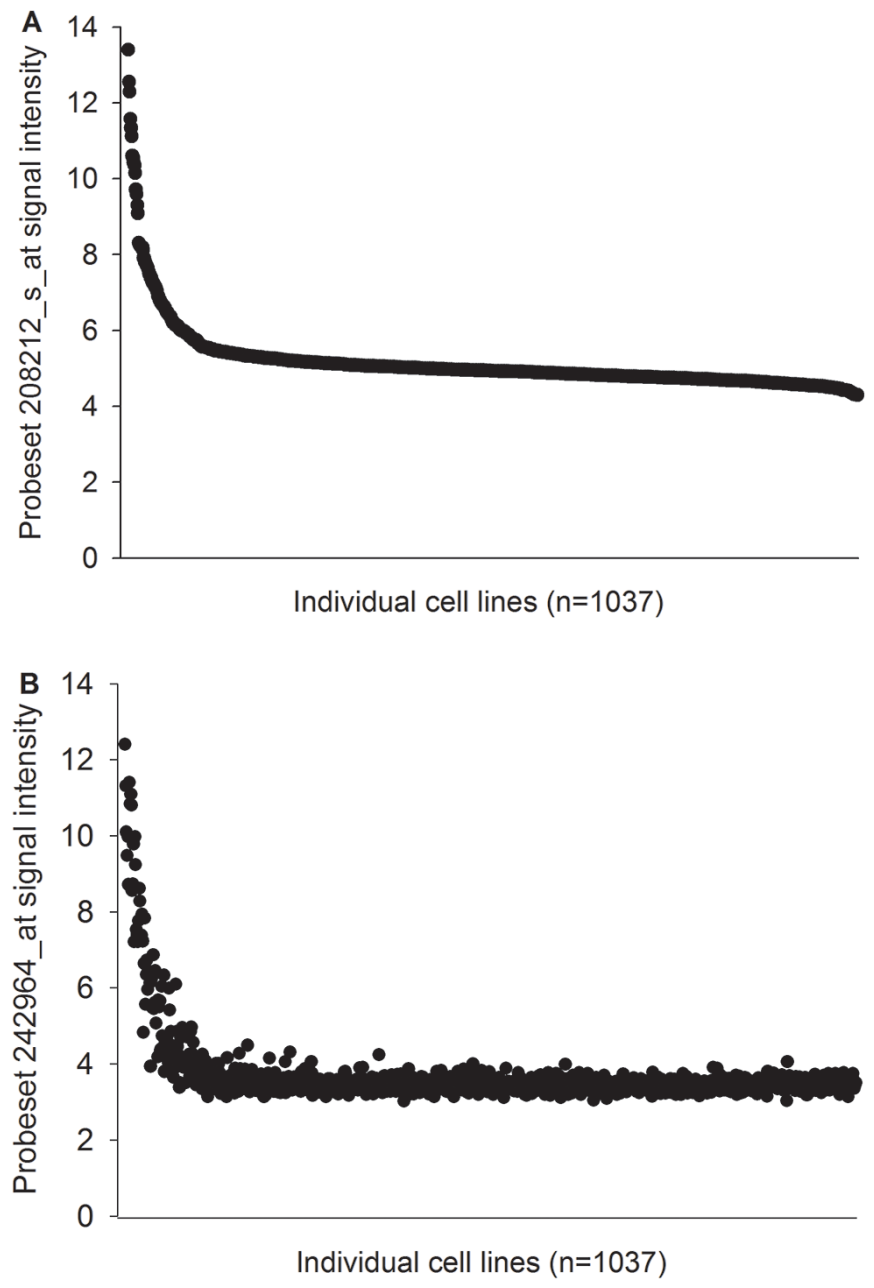

Figure 1: sweyjawbu expression is highly associated to ALK activated transcription. Probe sets 208212 s at (ALK) and 242964_at (sweyjawbu) signal intensities from 1037 different cell lines from Cancer Cell Line Encyclopedia (CCLE) database were plotted. Samples were arranged from highest to lowest by comparing the 208212 s_at probe set signal intensity. Those two probe sets' expression were highly correlated. 
The signal intensities from three lymphoma cell lines were extracted from the dataset for demonstration. Probe set 242964_at exhibited positive expression only in ALK rearrangement-positive cells, but was below background level in ALK rearrangement-negative cells (Figure 2A). Real-time PCR experiments also indicated that there was more than one hundred fold difference between ALK rearrangement-positive cells and ALK rearrangement-negative cells in sweyjawbu RNA expression level (Figure 2B).

\section{PCR assay detects ALK rearrangement status in lymphomas}

ALK rearrangement in lymphomas is relatively rare. There were 11 ALK rearrangement-positive ALCL patients and 1 ALK rearrangement positive DLBCL patient among the $13 \mathrm{ALCL}$ and 63 DLBCL patients used in our experiment. Comparable results were observed from PCR assay and IHC or FISH tests, to determine ALK rearrangement status in lymphomas. sweyjawbu gene expression differed significantly between ALK rearrangement positive lymphomas and ALK rearrangement-negative lymphomas. sweyjawbu also improved the discriminative power of the 5' and 3 ' portion ratio of the ALK transcript (Figure 3; Supplementary Table 2).

\section{RNA in situ hybridization localizes sweyjawbu and ALK in lymphomas}

RNAscope technology provides a revolutionary RNA in situ hybridization method to characterize RNA expression in single cells. Significant ALK kinase domain RNA expression was observed in ALK rearrangementpositive lymphoma cells. Simultaneously, ALK rearrangement positive lymphoma cells also expressed sweyjawbu RNA. In situ RNA hybridization confirmed the association of sweyjawbu and ALK (Figure 4).

\section{DISCUSSION}

With the rapid evolution of microarray technology over the last decade, multiple studies have characterized cancer cell lines and tumor tissue using standardized genome-wide microarray, and have generated large volumes of gene expression data. However, standard Affymetrix probe set annotation is at the gene level, and it has been reported that transcript-level annotation improves the interpretation of gene expression data
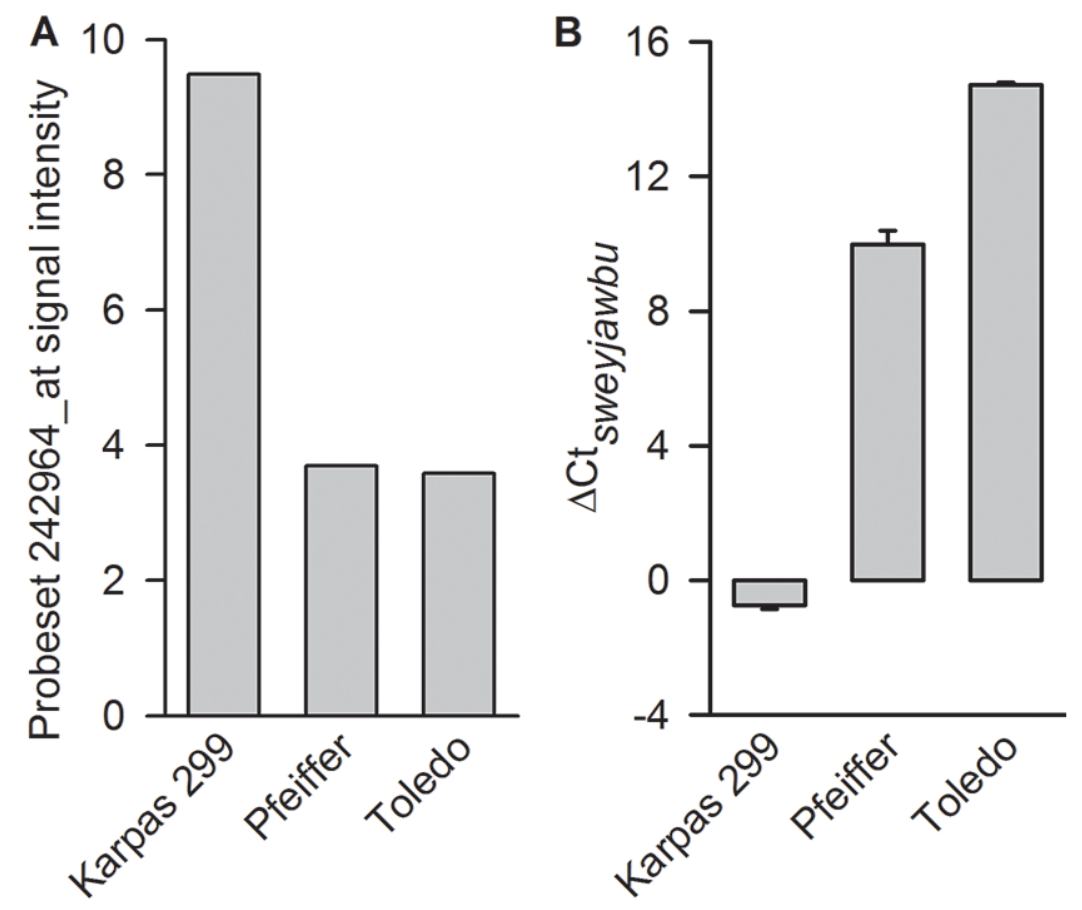

Figure 2: Differences were observed between ALK rearrangement-positive and ALK rearrangement-negative lymphoma cells. A. Compare probe set 242964_at signal intensity. B. Compare the relative expression $(\Delta \mathrm{Ct})$ value for sweyjawbu gene. Probe set 242964_at signal intensity was extracted from Cancer Cell Line Encyclopedia, www.broadinstitute.org/ccle. PCR experiments were performed in quadruplicate. There is no amplification for sweyjawbu gene in Toledo cell. Then the corresponding Ct value for sweyjawbu gene was set to 35 for result demonstration. 

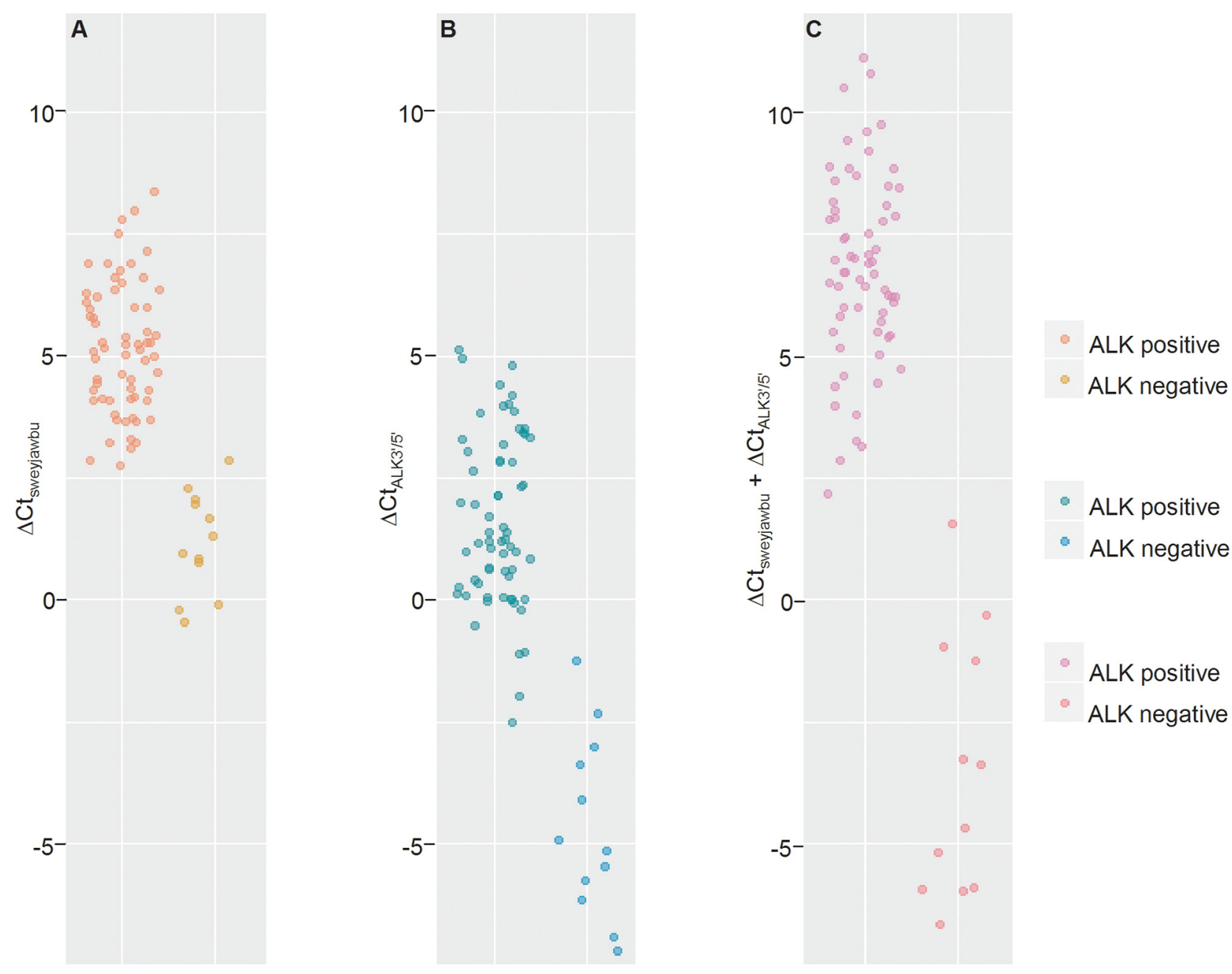

Figure 3: Differences were observed between ALK rearrangement-Positive lymphomas and ALK rearrangementnegative lymphomas. A. Compare $\Delta \mathrm{Ct}$ value for sweyjawbu gene. B. Compare $\Delta \mathrm{Ct}$ value for 5' portion and 3' portion of ALK transcript. C. Compare $\Delta \mathrm{Ct}$ value for sweyjawbu gene $+\Delta \mathrm{Ct}$ value for 5' portion and 3' portion of ALK transcript. 13 ALCL tumors and 63 DLBCL tumors were included into this study. The 11 ALCL and 1 DLBCL tumors confirmed as ALK-positive with FISH or IHC, exhibited lower $\Delta \mathrm{Ct}$ value. Combination of $\Delta \mathrm{Ct}_{\text {sweyjawbu }}$ and $\Delta \mathrm{Ct}_{\mathrm{ALK} 3 / 5 /}$, could distinguish ALK rearrangement positive samples from ALK rearrangementnegative samples, despite a few misclassifications when used separately.
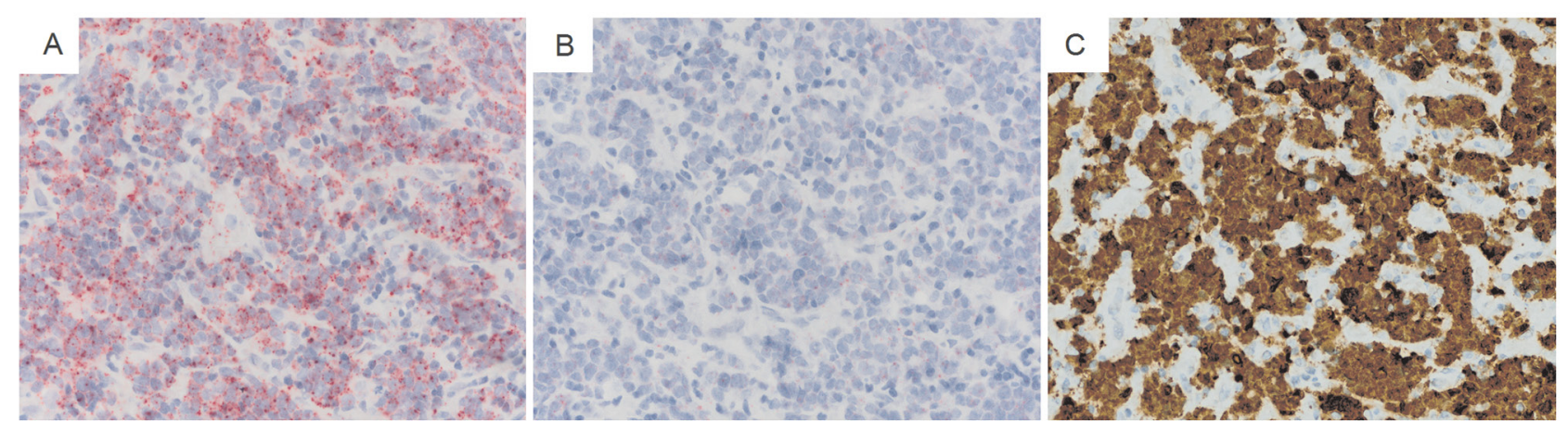

Figure 4: : In situ hybridization to characterize ALK RNA and sweyjawbu RNA expression in lymphoma cells. Intensive fast red Staining was observed in ALK rearrangement positive lymphoma cells. A. Section hybridized with probes target human ALK exon 19 to exon 29. B. Section hybridized with probes target human sweyjawbu. C. IHC was performed to determine ALK rearrangement status. 
[10]. In our experiment, we have aligned probe sequences from probe sets in traditional U133 Plus 2.0 gene expression arrays against the currently most comprehensive collection of transcript sequences from AceView database. Aid from this RNA level reannotation makes using publicly available microarray gene expression databases an economical and efficient way to perform other data-mining studies. For example, we identified a novel, non-coding RNA linked to ALK activation which had been recorded but had not been detailed by previous studies [11, 12].

There are several commercial tests to detect ALK rearrangements or hybrids [13]. FISH is a relatively wellstandardized but expensive method that requires biopsy. The interpretation of FISH results also requires specialized training due to considerable reader-related variability [14]. FISH results are affected by other factors such as long-duration specimen fixation, high temperature, and improper protein digestion. IHC is a relatively inexpensive method for diagnosing ALK rearranged lymphoma that, like FISH, requires biopsy sample. Variations in fusion partner may also influence protein levels and location [14]. Next generation sequencing provides the capability to detect multiple molecular alterations in a single assay. However, it may take several weeks to complete the experiment and data analysis [15] and the approach requires more technical manipulation, which might delay its clinical application.

In this study, a novel non-coding RNA sweyjawbu was identified from microarray datasets for prediction of ALK rearrangement. Previously, no report detailed the sweyjawbu gene, and its in vivo function is unknown yet. The ALK gene maps to chromosome 2, from 30144451 to 29415634 (NCBI 37, August 2010), on the reverse strand, while the sweyjawbu gene maps to chromosome 2, from 29414340 to 29413859 , also on the reverse strand. There was only a $1294 \mathrm{bp}$ distance between the two genes on the chromosome. sweyjawbu expression differed distinctly between ALK rearrangement-positive cells and ALK rearrangement-negative cells. RNAscope technology indicated both ALK kinase domain RNA and sweyjawbu RNA were co-expressed in lymphoma cells. Future studies will be undertaken to understand whether the sweyjawbu and ALK genes are transcriptionally coupled neighboring genes [16].

Highly comparable results can be generated from microarray and qRT-PCR platform [17]. Based on this discovery, combined with other published known methods, we have designed a PCR assay to detect ALK RNA hybrid status in both ALCL and DLBCL patient tissue samples. A significant advantage of the PCR-based assay is that it can be easily used in tissues obtained from minimally-invasive biopsy such as fine needle aspiration (FNA) or Cerebro-Spinal Fluid (CSF), which may not provide tissue suitable for preparation of FFPE samples [18]. The assay also could be complementary to FISH and IHC, which might further improve the accuracy and convenience of diagnosis and treatment of ALK rearrangement-positive lymphomas, or even other lymphomas of unknown origin with rare ALKpositive condition. Moreover, the prototype assay could be transferred to the FilmArray platform [19], which is an FDA-cleared multiplex PCR system that integrates sample preparation, amplification, detection and analysis in an hour. In summary, the new essay has the potential to change current clinical practice.

\section{MATERIALS AND METHODS}

\section{Cancer cell lines and clinical samples}

ALK rearrangement-positive cell line Karpas 299 (ALCL), and two ALK rearrangement-negative cell lines, Pfeiffer (DLBCL) and Toledo (DLBCL) were utilized. Karpas 299 cell line from Sigma-Aldrich was maintained in RPMI 1640 medium supplemented with $20 \%$ Fetal Bovine Serum (FBS) and 2 mM L-glutamine. ATCC cell lines, Pfeiffer and Toledo, were cultured in RPMI-1640 medium supplemented with 10\% FBS and $2 \mathrm{mM}$ L-glutamine. Cells were cultured with $6 \mathrm{~cm}$ dishes, and roughly $1 \times 10^{6}$ cells were collected with RNAprotect cell reagent (Qiagen). After homogenizing the lysate with QIAshredder spin column (Qiagen), the RNAs were extracted with RNeasy Plus mini kit (Qiagen).

Fresh frozen lymphoid tissues were obtained from 3 ALCL and 63 DLBCL patients for analysis. In addition, 10 Formalin-fixed and paraffin-embedded (FFPE) tissues prepared in the last 2 years, from ALK rearrangement positive ALCL patients were used. The median age of patients was 55 years old. There were more male patients (71\%) than female patients $(29 \%)$. Despite 11 patients with unknown status, there are more early stage patients (63\%) than advanced stage patients (37\%). Written informed consent was obtained from all participants. The study was approved by the Ethics Committee of Fudan University Shanghai Cancer Center, China, and the procedures were conducted according to the principles expressed in the Declaration of Helsinki. Detailed clinical information is provided in Supplementary Table 2. Frozen tissue resected from lymphoma patients were homogenized in TRIZOL using mortar and pestle in the presence of liquid nitrogen. RNAs were extracted with RNAeasy Plus mini kit (Qiagen). FFPE tissues were deparaffinized with xylene and RNA was subsequently extracted with RecoverAll Total Nucleic Acid Isolation Kit (ThermoFisher Scientific).

The RNA quality was checked by electrophoresis of total RNA, followed by staining with ethidium bromide. The absorbance of each RNA at 260nm and 280nm were measured using a spectrophotometer for RNA quantity and purity. 


\section{Primer design}

The primer pairs were designed using the Beacon Designer software (Premier Biosoft, Palo Alto, CA). Primers' sequences were compared with UCSC SNP database for known single-nucleotide polymorphism (SNP) information. The final concentration of each primer in the reaction tube was $0.3 \mu \mathrm{M}$. Primer pairs with efficient amplification and single melt curves were kept for further analysis. Detailed primer sequences were provided in Supplementary Table 1.

\section{Real-time PCR detection}

To detect templates from cell lines or tissue samples, cDNA was synthesized from $1 \mu \mathrm{g}$ RNA with QuantiTect Reverse Transcription kit (Qiagen). Quantitative realtime PCR detection was performed on an ABI 7900HT instrument using QuantiFast SYBR Green PCR kit (Qiagen). After initial activation for $5 \mathrm{~min}$ at $95^{\circ} \mathrm{C}$, twostep cycling was utilized for 40 cycles with $10 \mathrm{~s}$ at $95^{\circ} \mathrm{C}$ and $30 \mathrm{~s}$ at $60^{\circ} \mathrm{C}$, followed by a melting curve analysis. Gene expression was normalized by TB and PGK genes in lymphomas [20].

\section{RNAscope RNA in situ hybridization study}

Advanced Cell Diagnostics (ACD) Company introduced RNAscope technology for RNA in situ hybridization. Standard $35 \mathrm{ZZ}$ probes targeting human ALK exon 19 to exon 29 were used to characterize ALK RNA 3' end region. Custom designed $8 \mathrm{ZZ}$ probes targeting sweyjawbu were used. Continuous FFPE sections were hybridized with dedicated probes and detected with fast red dye.

\section{Patients' ALK rearrangement status confirmation}

Selected patients' ALK rearrangement status were checked either by FISH (Abbott), or IHC (VENTANA ALK (D5F3) CDx Assay, Roche) by the Department of Pathology, Fudan University Shanghai Cancer Center. FISH-positive cases were defined as two positive ALK rearrangement patterns. Positive cases were defined as more than $15 \%$ strand-break signals or isolated red signal signals in 50 tumor cells. IHC staining was scored by two pathologists as follows: 0 (no staining); $1+$ (faint, cytoplasmic staining); 2+ (moderate, smooth cytoplasmic staining); $3+$ (intense, granular cytoplasmic staining) in more than $10 \%$ of tumor cells. Anti-ALK IHC staining results were interpreted using a binary scoring system: positive $(3+$ or $2+)$ or negative $(1+$ or 0$)$.

\section{ACKNOWLEDGMENTS}

We would like to thank Ping Zhao (Department of Biology, University of North Alabama), M.D., Ph.D., for assistance with editing the article. This study was supported by grants from National Natural Science Foundation of China (no. 81400161).

\section{CONFLICTS OF INTEREST}

The authors declare no conflicts of interest.

\section{REFERENCES}

1. Gisselbrecht C, Schmitz N, Mounier N, Singh Gill D, Linch DC, Trneny M, Bosly A, Milpied NJ, Radford J, Ketterer N, Shpilberg O, Duhrsen U, Hagberg H, et al. Rituximab maintenance therapy after autologous stem-cell transplantation in patients with relapsed CD20(+) diffuse large B-cell lymphoma: final analysis of the collaborative trial in relapsed aggressive lymphoma. J Clin Oncol. 2012; 30:4462-4469.

2. Siegel R, Naishadham D and Jemal A. Cancer statistics, 2013. CA Cancer J Clin. 2013; 63:11-30.

3. Morris SW, Kirstein MN, Valentine MB, Dittmer K, Shapiro DN, Look AT and Saltman DL. Fusion of a kinase gene, ALK, to a nucleolar protein gene, NPM, in non-Hodgkin's lymphoma. Science. 1995; 267:316-317.

4. Minoo P and Wang HY. ALK-immunoreactive neoplasms. Int J Clin Exp Pathol. 2012; 5:397-410.

5. Abramson JS and Zelenetz AD. Recent advances in the treatment of non-Hodgkin's lymphomas. J Natl Compr Canc Netw. 2013; 11:671-675.

6. Kwak EL, Bang YJ, Camidge DR, Shaw AT, Solomon B, Maki RG, Ou SH, Dezube BJ, Janne PA, Costa DB, VarellaGarcia M, Kim WH, Lynch TJ, et al. Anaplastic lymphoma kinase inhibition in non-small-cell lung cancer. N Engl J Med. 2010; 363:1693-1703.

7. Li J, Ouyang J, Zhou R, Chen B and Xu Y. Promising response of anaplastic lymphoma kinase-positive large B-cell lymphoma to crizotinib salvage treatment: case report and review of literature. Int J Clin Exp Med. 2015; 8:6977-6985.

8. Mitou G, Frentzel J, Desquesnes A, Le Gonidec S, AlSaati T, Beau I, Lamant L, Meggetto F, Espinos E, Codogno P, Brousset $\mathrm{P}$ and Giuriato $\mathrm{S}$. Targeting autophagy enhances the anti-tumoral action of crizotinib in ALK-positive anaplastic large cell lymphoma. Oncotarget. 2015; 6:3014930164. doi: 10.18632/oncotarget.4999.

9. Barretina J, Caponigro G, Stransky N, Venkatesan K, Margolin AA, Kim S, Wilson CJ, Lehár J, Kryukov GV and Sonkin D. The Cancer Cell Line Encyclopedia enables 
predictive modelling of anticancer drug sensitivity. Nature. 2012; 483:603-607.

10. Yu H, Wang F, Tu K, Xie L, Li Y-Y and Li Y-X. Transcriptlevel annotation of Affymetrix probesets improves the interpretation of gene expression data. BMC bioinformatics. 2007; 8:1.

11. Okayama H, Kohno T, Ishii Y, Shimada Y, Shiraishi K, Iwakawa R, Furuta K, Tsuta K, Shibata T and Yamamoto $\mathrm{S}$. Identification of genes upregulated in ALK-positive and EGFR/KRAS/ALK-negative lung adenocarcinomas. Cancer research. 2012; 72:100-111.

12. Piva R, Agnelli L, Pellegrino E, Todoerti K, Grosso V, Tamagno I, Fornari A, Martinoglio B, Medico E and Zamò A. Gene expression profiling uncovers molecular classifiers for the recognition of anaplastic large-cell lymphoma within peripheral T-cell neoplasms. Journal of clinical oncology. 2010; 28:1583-1590.

13. Leighl NB, Rekhtman N, Biermann WA, Huang J, MinoKenudson M, Ramalingam SS, West H, Whitlock S and Somerfield MR. Molecular testing for selection of patients with lung cancer for epidermal growth factor receptor and anaplastic lymphoma kinase tyrosine kinase inhibitors: American Society of Clinical Oncology endorsement of the College of American Pathologists/International Association for the study of lung cancer/association for molecular pathology guideline. J Clin Oncol. 2014; 32:3673-3679.

14. Wallander ML, Geiersbach KB, Tripp SR and Layfield LJ. Comparison of reverse transcription-polymerase chain reaction, immunohistochemistry, and fluorescence in situ hybridization methodologies for detection of echinoderm microtubule-associated proteinlike 4-anaplastic lymphoma kinase fusion-positive non-small cell lung carcinoma: implications for optimal clinical testing. Arch Pathol Lab Med. 2012; 136:796-803.

15. Steinhilber J, Bonin M, Walter M, Fend F, Bonzheim I and Quintanilla-Martinez L. Next-generation sequencing identifies deregulation of microRNAs involved in both innate and adaptive immune response in ALK+ ALCL. PLoS One. 2015; 10:e0117780.

16. De $\mathrm{S}$ and Babu MM. Genomic neighbourhood and the regulation of gene expression. Current opinion in cell biology. 2010; 22:326-333.

17. Xu Y, Xu Q, Yang L, Ye X, Liu F, Wu F, Ni S, Tan C, Cai G and Meng $\mathrm{X}$. Identification and validation of a blood-based 18-gene expression signature in colorectal cancer. Clinical Cancer Research. 2013; 19:3039-3049.

18. Stoos-Veic T, Livun A, Ajdukovic R, Pejsa V, Jaksic O and Kusec R. Detection of $t(14 ; 18)$ by PCR of IgH/BCL2 fusion gene in follicular lymphoma from archived cytological smears. Coll Antropol. 2010; 34:425-429.

19. Poritz MA, Blaschke AJ, Byington CL, Meyers L, Nilsson $\mathrm{K}$, Jones DE, Thatcher SA, Robbins T, Lingenfelter B and Amiott E. FilmArray, an automated nested multiplex PCR system for multi-pathogen detection: development and application to respiratory tract infection. PloS one. 2011; 6:e26047.

20. Lossos I, Czerwinski D, Wechser M and Levy R. Optimization of quantitative real-time RT-PCR parameters for the study of lymphoid malignancies. Leukemia. 2003; 17:789-795. 